\title{
Correction to: One-sided invertibility of discrete operators with bounded coefficients
}

\author{
Luis Eduardo Flores-Zapotitla and Yuri I. Karlovich(D
}

Correction to: Aequat. Math. https://doi.org/10.1007/s00010-020-00773-8

\section{Addendum}

By the sufficiency in Theorem 3.12, the left (resp., right) invertibility of the operators $A \in\left\{A_{E}, \widetilde{A}_{E}, A_{E_{m}}, \widetilde{A}_{E_{m}}\right\}$ on the spaces $l^{p}(1<p<\infty)$ follows from the invertibility of the operator $A^{\diamond} A$ (resp., $A A^{\diamond}$ ). The Fredholmness of such operators $A$ is not necessary for their one-sided invertibility (for example, the right invertible operator $A_{E_{m}}=E_{m}$ is not Fredholm for $|m|>1$ ). On the other hand, the invertibility criterion for the operators $B \in\left\{A^{\diamond} A, A A^{\diamond}\right\}$ presented in Theorem 3.15 is also valid if $B \in \mathcal{W}_{p}$ and $A \notin \mathcal{W}_{p}$.

As a result, Theorems 4.4, 4.5, 5.4, 5.5 can be essentially reinforced by excluding the Fredholm condition for the operators $A_{E}, \widetilde{A}_{E}, A_{E_{m}}, \widetilde{A}_{E_{m}}$, respectively, and applying the invertibility criterion from Theorem 3.15.

The modified theorems have the following form.

Theorem 6.1. The operator $A_{E}$ given by (4.2) and satisfying (4.3) is left invertible on the space $l^{p}$ for $p \in(1, \infty)$ if for $B=A_{E}^{\diamond} A_{E}$ and all sufficiently large $n \in \mathbb{N}$ the operators $B_{n}^{ \pm}=P_{n}^{ \pm} B P_{n}^{ \pm}$are invertible on the spaces $P_{n}^{ \pm} l^{p}$, respectively, and the $(2 n-1) \times(2 n-1)$ matrix $B_{n, 0}$ defined for $B=A_{E}^{\diamond} A_{E}$ by (3.33) is invertible. The operator $A_{E}$ given by (4.2) and satisfying (4.3) and condition (A) is right invertible on the space $l^{p}$ for $p \in(1, \infty)$ if for $B=A_{E} A_{E}^{\diamond}$ and all sufficiently large $n \in \mathbb{N}$ the operators $B_{n}^{ \pm}=P_{n}^{ \pm} B P_{n}^{ \pm}$are invertible on the spaces $P_{n}^{ \pm} l^{p}$, respectively, and the $(2 n-1) \times(2 n-1)$ matrix $B_{n, 0}$ defined

The original article can be found online at https://doi.org/10.1007/s00010-020-00773-8. 
for $B=A_{E} A_{E}^{\diamond}$ by (3.33) is invertible. Under these conditions, one of the left (resp., right) inverses of the operator $A_{E}$ is given by $A_{E}^{L}=\left(A_{E}^{\diamond} A_{E}\right)^{-1} A_{E}^{\diamond}$ (resp., by $\left.A_{E}^{R}=A_{E}^{\diamond}\left(A_{E} A_{E}^{\diamond}\right)^{-1}\right)$.

Theorem 6.2. The operator $\widetilde{A}_{E}$ given by (4.2) and satisfying (4.3) is left invertible on the space $l^{p}$ for $p \in(1, \infty)$ if for $B=\widetilde{A}_{E}^{\diamond} \widetilde{A}_{E}$ and all sufficiently large $n \in \mathbb{N}$ the operators $B_{n}^{ \pm}=P_{n}^{ \pm} B P_{n}^{ \pm}$are invertible on the spaces $P_{n}^{ \pm} l^{p}$, respectively, and the $(2 n-1) \times(2 n-1)$ matrix $B_{n, 0}$ defined for $B=\widetilde{A}_{E}^{\diamond} \widetilde{A}_{E}$ by (3.33) is invertible. The operator $\widetilde{A}_{E}$ given by (4.2) and satisfying (4.3) and condition $(\mathrm{B})$ is right invertible on the space $l^{p}$ for $p \in(1, \infty)$ if $E$ is a permutation operator and for $B=\widetilde{A}_{E} \widetilde{A}_{E}^{\diamond}$ and for all sufficiently large $n \in \mathbb{N}$ the operators $B_{n}^{ \pm}=P_{n}^{ \pm} B P_{n}^{ \pm}$are invertible on the spaces $P_{n}^{ \pm} l^{p}$, respectively, and the $(2 n-1) \times(2 n-1)$ matrix $B_{n, 0}$ defined for $B=\widetilde{A}_{E} \widetilde{A}_{E}^{\diamond}$ by $(3.33)$ is invertible. Under these conditions, one of the left (resp., right) inverses of the operator $\widetilde{A}_{E}$ is given by $\widetilde{A}_{E}^{L}=\left(\widetilde{A}_{E}^{\diamond} \widetilde{A}_{E}\right)^{-1} \widetilde{A}_{E}^{\diamond}\left(\right.$ resp. , by $\left.\widetilde{A}_{E}^{R}=\widetilde{A}_{E}^{\diamond}\left(\widetilde{A}_{E} \widetilde{A}_{E}^{\diamond}\right)^{-1}\right)$.

Theorem 6.3. Let $p \in(1, \infty)$ and $m \in \mathbb{Z} \backslash\{0\}$. Then the slant-dominated discrete Wiener-type operator $A_{E_{m}}$ is left (resp., right) invertible on the space $l^{p}$ if for $B=A_{E_{m}}^{\diamond} A_{E_{m}}$ (resp., for $B=A_{E_{m}} A_{E_{m}}^{\diamond}$ ) and for all sufficiently large $n \in \mathbb{N}$ the operators $B_{n}^{ \pm}=P_{n}^{ \pm} B P_{n}^{ \pm}$are invertible on the spaces $P_{n}^{ \pm} l^{p}$, respectively, and the $(2 n-1) \times(2 n-1)$ matrix $B_{n, 0}$ given by $(3.33)$ for $B=$ $A_{E_{m}}^{\diamond} A_{E_{m}}$ (resp., for $B=A_{E_{m}} A_{E_{m}}^{\diamond}$ ) is invertible. Under these conditions, one of the left (resp., right) inverses of the operator $A_{E_{m}}$ is given by $A_{E_{m}}^{L}=$ $\left(A_{E_{m}}^{\diamond} A_{E_{m}}\right)^{-1} A_{E_{m}}^{\diamond}$ (resp., by $\left.A_{E_{m}}^{R}=A_{E_{m}}^{\diamond}\left(A_{E_{m}} A_{E_{m}}^{\diamond}\right)^{-1}\right)$.

Theorem 6.4. Let $p \in(1, \infty)$ and $m \in \mathbb{Z} \backslash\{0\}$. Then the slant-dominated discrete Wiener-type operator $\widetilde{A}_{E_{m}}$ is left (resp., right) invertible on the space $l^{p}$ if for $B=\widetilde{A}_{E_{m}}^{\diamond} \widetilde{A}_{E_{m}}$ (resp., for $B=\widetilde{A}_{E_{m}} \widetilde{A}_{E_{m}}^{\diamond}$ ) and for all sufficiently large $n \in \mathbb{N}$ the operators $B_{n}^{ \pm}=P_{n}^{ \pm} B P_{n}^{ \pm}$are invertible on the spaces $P_{n}^{ \pm} l^{p}$, respectively, and the $(2 n-1) \times(2 n-1)$ matrix $B_{n, 0}$ given by $(3.33)$ for $B=$ $\widetilde{A}_{E_{m}}^{\diamond} \widetilde{A}_{E_{m}}$ (resp., for $B=\widetilde{A}_{E_{m}} \widetilde{A}_{E_{m}}^{\diamond}$ ) is invertible, where $|m|=1$ in the case of right invertibility. Under these conditions, one of the left (resp., right) inverses of the operator $\widetilde{A}_{E_{m}}$ is given by $\widetilde{A}_{E_{m}}^{L}=\left(\widetilde{A}_{E_{m}}^{\diamond} \widetilde{A}_{E_{m}}\right)^{-1} \widetilde{A}_{E_{m}}^{\diamond}$ (resp., by $\widetilde{A}_{E_{m}}^{R}=$ $\left.\widetilde{A}_{E_{m}}^{\diamond}\left(\widetilde{A}_{E_{m}} \widetilde{A}_{E_{m}}^{\diamond}\right)^{-1}\right)$.

Publisher's Note Springer Nature remains neutral with regard to jurisdictional claims in published maps and institutional affiliations. 
Luis Eduardo Flores-Zapotitla and Yuri I. Karlovich

Centro de Investigación en Ciencias, Instituto de Investigación en Ciencias Básicas y Aplicadas

Universidad Autónoma del Estado de Morelos

Av. Universidad 1001, Col. Chamilpa

C.P. 62209 Cuernavaca Morelos

Mexico

e-mail: luis.flores@uaem.edu.mx

Yuri I. Karlovich

e-mail: karlovich@uaem.mx 